\title{
State of the Art in \\ Skinning Techniques for Articulated Deformable Characters
}

\author{
Nadine Abu Rumman ${ }^{1}$ and Marco Fratarcangeli ${ }^{2}$ \\ ${ }^{1}$ Sapienza University of Rome, Rome, Italy \\ ${ }^{2}$ Chalmers University of Technology, Gothenburg, Sweden \\ aburumman@dis.uniroma1.it,marcof@chalmers.se
}

\begin{abstract}
Keywords: character animation, skinning, skeleton-based animation, physics-based animation
Abstract: $\quad$ Skinning is an indispensable component of the content creation pipeline for character animation in the context of feature films, video games, and in the special effects industry. Skinning techniques define the deformation of the character skin for every animation frame according to the current state of skeletal joints. In this state of the art report, we focus on the existing research in the areas of skeleton-based deformation, volume preserving techniques and physically based skinning methods. We also summarize the recent research in deformable and soft bodies simulations for articulated characters, and discuss various geometric and examples-based approaches.
\end{abstract}

\section{INTRODUCTION}

In character animation, skinning is the process of defining how the geometric surface of the character deforms according to a function of the skeletal poses. Creating believable and compelling skin deformations is one of the main challenges of animated feature films, computer games, and interactive applications. Traditionally, bone transformations describe the position and orientation of the joints, and the skin deformation is computed by linearly blending bone transformations to the skin. This technique is called "skeletal subspace deformation", also known as linear blend skinning (LBS), and it has been described for the first time in (MagnenatThalmann et al., 1988). However, such simple and linear blending to the bone transformations cannot be expected to capture complex deformations. Moreover, because the deformation is restricted to the subspace of the affine transformation of the bones, this method has problems deforming the skin near joints due to volume loss or the well-known "candy-wrapper" artefacts. By replacing linear blending with a nonlinear blending (dual quaternion skinning, (Kavan et al., 2007)), the artefacts of LBS can be completely avoided. However, dual quaternion blending suffers from an undesirable joint-bulging artefact while bending, which requires artistic manual work to be fixed. Although these methods achieve real-time performance, they are purely kinematic lacking of secondary motion effects, such as jiggling motion of fatty tissues and contact deformation effects. In contrast, by employing a physically based method into the skinning process, the believability of character motions is highly enhanced. Physicsbased simulations manage to bring skeleton-based animation beyond the purely kinematic approach by simulating secondary motions like jiggling, volume preservation and contact deformation effects. These secondary motions enrich the visual experience of the animation and are essential for creating appealing characters animation. Despite offering such realistic effects, physically based simulation is computationally demanding and complex, thus it is usually avoided in interactive applications. Furthermore, physically based methods require human intervention to generate input data, which describe the physical states, before the problem can be solved by a computer program. In most cases, such preparation is tedious and the artist must master the knowledge of both the given software and the underlying physics of the phenomenon. The key challenge of producing believable deformations is to satisfy the conflicting requirements of real-time interactivity and believability. Believability requires achieving sufficient deformation detail, which means capturing the full range of desired effects, namely jiggling, volume preservation, muscle bulging and skin contact deformations. Producing these deformations demand at least an order of magnitude more computation time than current interactive deformation systems. 
The aim of this paper is to provide a comprehensive survey on the existing skinning techniques in literature. These can be classified into three categories: skeleton-based deformation, volume preserving skinning and physics-based skinning methods. We discuss the existing skinning methods and how they address the above-mentioned problems, and we also highlight the advantages and disadvantages of each method. Skinning methods can be also classified into smooth skinning, data-driven skinning, and physics-based skinning.

\section{SKELETON-BASED SKINNING METHODS}

The most common approach for deforming articulated character' skin is to define the surface geometry as a function of an underlying skeletal structure. Due to the simplicity, intuitive manipulation, and the ability to quickly solve inverse-kinematics on a small subspace (i.e., the skeleton), skeleton-based methods are very popular and widely used in the animation industry (Le and Deng, 2012). When modeling a skeleton-based deformation, the challenge is to obtain high-quality skin deformations in real-time given an arbitrary skeletal posture. The current skeletonbased deformation techniques can be divided into two sections: geometry-based methods (Section 2.1) and example-based methods (Section 2.2).

\subsection{Geometric Skinning Techniques}

In geometric skinning techniques, skeleton-to-skin binding is defined in a direct, geometrical way. Geometric approaches to deform articulated characters have shown reasonable results at interactive rates. We start by discussing the standard real-time method "skeletal subspace deformation", also known as linear blend skinning (LBS) presented in (MagnenatThalmann et al., 1988). This method has been widely adopted in real-time applications such as games, for its computational efficiency and straightforward GPU implementation. Unfortunately, linear blend skinning suffers from visual artefacts like self intersection, volume loss or the well-known "candy-wrapper" $\operatorname{artefact}^{1}$ (see Fig. 1), which are the result of the linear nature of the algorithm, since the linear interpolation of the transformation matrices is not equivalent to the linear interpolation of their rotations

\footnotetext{
${ }^{1}$ the "candy-wrapper" artefact is the skin collapsing effect exhibited by linear blend skinning (MagnenatThalmann et al., 1988).
}

(Alexa, 2002). The limitations of LBS have been extensively studied, where many techniques have been proposed to avoid its artefacts. One possibility is to enrich the space of skinning weights, leading to methods which are still linear but offer a wider range of deformations. These methods are called multi-linear skinning techniques (Wang and Phillips, 2002; Mohr and Gleicher, 2003), in which the extra weights are learned from input examples and regularization is used to prevent overfitting. Merry et al. propose a multi-linear skinning model called Animation Space (Merry et al., 2006), which uses 4 weights per vertex-bone pair. However, this increase in the number of weights carries an additional cost in time and space, as well as parameter passing. While linear skinning techniques are popular due to their efficient implementations, they cannot entirely remove the "candy-wrapper" artefact, which is in all cases noticeable under large joint rotations. For a comprehensive survey on linear skinning techniques, we refer the interested reader to (Jacka et al., 2007). Selecting good skinning weights is critical to avoid the artefacts and generate more natural deformations. Recently, an automatic computation of skinning weights was presented in (Dionne and de Lasa, 2013). In their method, the influence weights are determined using geodesic distances from each bone, which makes the inverse-distance weights shapeaware and can even work with production meshes that may contain non-manifold geometry. Despite that associating skinning weights with the mesh vertices can be done automatically, this method tends to either increase or decrease the volume around joints.

By replacing linear blending with nonlinear blending (Hejl, 2004; Kavan and Zára, 2005; Kavan et al., 2007), the "candy-wrapper" artefact can be completely avoided. Nonlinear skinning methods convert the affine rigid transformation matrices to $<$ quaternion,translation $>$ pairs, which are then easier to blend than their matrix equivalents (Hejl, 2004; Kavan and Zára, 2005). In (Hejl, 2004) some constraints are imposed on the character's rigging, whereas spherical skinning (Kavan and Zára, 2005) uses a computationally expensive Singular Value Decomposition (SVD) scheme. The practical impact of these two methods is limited because of their dealing with the translational component of the skinning transformations. In contrast, dual quaternion skinning (DQS (Kavan et al., 2007)) uses an approximate blending technique based on dual quaternions (essentially, two regular quaternions). Dual quaternion skinning is able to achieve comparable speeds to LBS while increasing the visual 


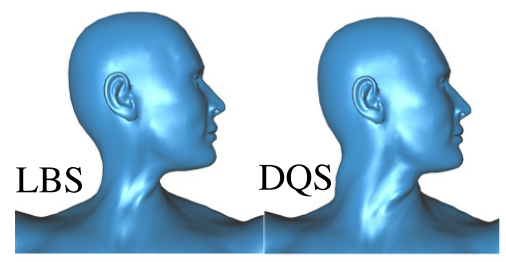

(a)

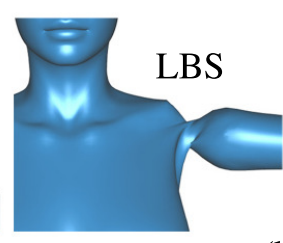

(b)

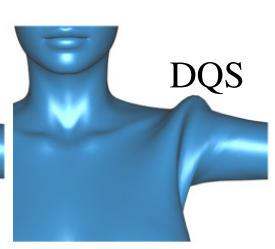

)

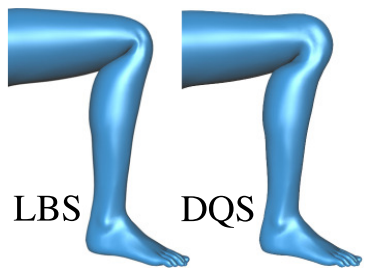

(c)

Figure 1: Demonstrates the artefacts of classic interactive skinning techniques linear blend skinning (LBS) and dual quaternion skinning (DQS). Linear blend skinning (LBS) is the most widely employed skinning technique, due to its simplicity and efficiency. Unfortunately, LBS suffers from the "candy-wrapper" artefact while twisting ((a) and (b)). This artefact can be eliminated by a nonlinear blending method such as dual quaternion skinning (DQS), but DQS produces an unnatural jointbulging artefact while bending (c). Observing that LBS does not produce bulging while bending and DQS does not suffer from the "candy-wrapper" artefact while twisting. (Image taken from (Kavan and Sorkine, 2012).)

quality of the animation. However, it suffers from an undesired joint bulging ${ }^{2}$ artefact (as shown in Fig. 1), which requires artistic manual work to be fixed. Manually fixing these artefacts is a a tedious process, so automatic skinning techniques are becoming increasingly popular (Baran and Popović, 2007; Kavan et al., 2009; Chen et al., 2011; Jacobson et al., 2011; Bharaj et al., 2012; Jacobson et al., 2012). Moreover, an interesting extension of linear blend skinning called spline-skinning comes from (Yang et al., 2006; Forstmann and Ohya, 2006; Forstmann et al., 2007), which often produces better skinning deformations and suppresses (but not completely eliminates) the "candy wrapper" artefact. Instead of using conventional matrix rotations, spline-skinning represents each bone of the skeleton by a spline. Furthermore, an appealing extension of DQS that is successfully applied in a production setting (Disney's Frozen), is depicted in (Lee et al., 2013). For a extensive discussion on nonlinear skinning methods, we refer the reader to (Kavan et al., 2009). Whilst all the above-mentioned methods fully define the surface positions based on skeletal configuration, they cannot capture secondary motion effects and skin contact behavior in response to collision. Recently, more advanced geometric skinning methods were introduced to limit the artefacts of LBS, while keeping their simplicity. Kavan and Sorkine (Kavan and Sorkine, 2012) developed a new skinning method based on the concept of joint-based deformers, which avoids the artefacts of linear blend skinning as well as the bulging artefact of dual quaternion skinning. Jacobson et al. (Jacobson and Sorkine, 2011) expanded skinning to support bending, stretching and twisting by using a slight variation on the standard skinning equations. Impressive skinning results

\footnotetext{
2 joint-bulging is an unnatural skin bulging effect produced by dual quaternion skinning (Kavan et al., 2007) while bending.
}

can be obtained using the technique presented in (Vaillant et al., 2013), which generates visually plausible skin deformations in real-time. Their method automatically captures contact surfaces between skin parts without requiring any collision detection step. Moreover, they extended their framework to handle local skin contacts and produce the effect of skin elasticity (sliding effect) (Vaillant et al., 2014). More recently, (Kim and Han, 2014) proposed a post processing method for dual quaternion skinning, which eliminates the joint-bulging artefacts and its suitable for real-time character animation. In spite of improvements, geometric skinning techniques remains purely kinematic, lacking of secondary motions effects like passive jiggling motion of the fatty tissues or muscle bulging. In the next section, we present the most influential example-based skinning methods which are able to alleviate the limitations of geometric skinning method and add dynamic effects to the skin. For a more thorough treatment of these methods, we refer the reader to (Jacobson et al., 2014).

\subsection{Example-based Skinning Methods}

This class of skinning methods can be also called data-driven methods. In contrast to geometric approaches, example-based skinning methods permit more complex skinning effects such as muscle bulges and wrinkles, while also addressing the artefacts of linear skinning techniques. These methods take as input a series of sculpted example poses and interpolate them to obtain the desired deformation. One of the first example-based methods is pose space deformation (PSD, (Lewis et al., 2000)), which uses a radial basis function to interpolate correction vectors among the example poses. In pose space deformation method, pose space is a set of degrees of freedom of a character's model which vary between the example poses. A particular pose is a particular 


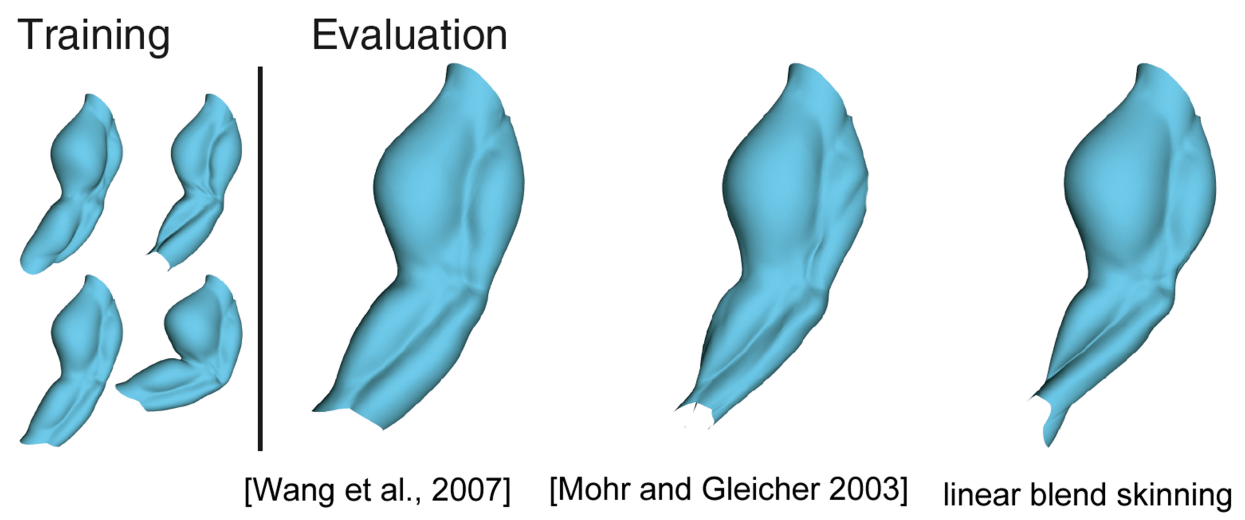

Figure 2: A set of example poses from an anatomically motivated arm model with both bending and twisting at the elbow. The twisting and muscle bulges are enough to prevent LBS from approximating the examples well. The technique of (Mohr and Gleicher, 2003) does better, but still differ from the given example poses. The model from (Wang et al., 2007) wellapproximate the examples poses. (Image taken from (Wang et al., 2007).)

configuration of these degrees of freedom. A more sophisticated extension of PSD was presented in (Sloan et al., 2001). Their method interpolates an articulated character using example poses scattered in an abstract space. This abstract space consists of dimensions describing global properties of the 3D character, such as age and gender, in addition to dimensions that are used to describe the configuration, such as the amount of bend at the elbow joint. Moreover, PSD was generalized to support weight in a technique called weighted pose space deformation (WPSD), in (Kurihara and Miyata, 2004; Rhee et al., 2006)), which largely reduces the number of required example poses. Although WPSD can handle large-scale deformations well, it cannot provide detailed deformation and it requires more computation than the original pose space deformation (PSD). In example-based methods, the amount of memory grows with the number of training examples, thus they are more popular in animated feature film (DreamWorks Animation's Shrek 2) than in real-time application. To tackle this problem, (Kry et al., 2002) proposed a method similar in spirit to PSD called EigenSkin. Instead of using all the displacements for example poses, they used precomputed principal components of deformation influences on individual joints. The resulting algorithm leads to considerable memory savings and enables to transfer the computations to the GPU. Despite the fact that pose space deformation methods are simple to implement, they require tremendous effort from artists because they have to create different poses by hand for a wide variety of examples. Another class of example-based methods, which is a direct generalization of LBS but does not require data interpolation, is composed by methods such as single-weight enveloping
(SWE, (Mohr and Gleicher, 2003)) and multi weight enveloping (MWE, (Wang and Phillips, 2002)). Single-weight enveloping estimated single weight per vertex with rigid character bones, with provisions made for adding additional bones. Multi-weight enveloping, instead, is based on a linear framework supporting multiple weights per vertex-bone, and it provides better approximations than SWE but at the cost of 12 weights per vertex-bone instead of 1 weight per vertex-bone as in SWE. However, linearity has certain benefits: it is fast and it can be used to derive a measure of average distance across the space of poses but the example meshes are still necessary in order to obtain the weights. This class of methods allows a smaller number of poses to be used to generate a larger number of deformations. On the other hand, they introduce an increased number of weight parameters which must be computed in a complex process.

As an alternative to using sculpted example poses, several example-based approaches use scanned or photographed data. Early work that uses 3D scanned poses of a human body in character skinning has been presented in (Min et al., 2000). Additionally, the method in (Allen et al., 2002) creates a high quality posable upper body model from range scan data and markers. In their method, they obtain deformations corresponding to different poses by matching a subdivision surface template to the range data. Recently, more advanced example-based techniques have been effectively integrated with mesh deformation algorithms to further improve the quality of skinning (Wang et al., 2007; Shi et al., 2008; Schumacher et al., 2012; Le and Deng, 2014). A rotational regression model is proposed 
in (Wang et al., 2007), which captures common skinning deformation such as muscle bulging (as we can see in Fig. 2) and twisting, specifically in challenging regions such as the shoulders. Park and Hodgins also introduced an interesting technique that captures and synthesizes detailed skin deformations such as bulging and jiggling (Park and Hodgins, 2006; Park and Hodgins, 2008), when a character performs dynamic activities. They use a very dense and large set of markers to capture the dynamic motions. Then, they employ a second-order skinning scheme followed by a radial basis function of the residual errors to provide detailed skin deformations. While high-quality skin deformations can be captured accurately using scanned data, marker-based motion capture systems typically have a time-consuming calibration process and significant hardware and set-up costs. Example-based skinning methods are attractive because they can provide rich details from physical measurements and add realistic secondary deformation to the skeleton-based animations. Shi et al. presented an appealing method that is able to provide the jiggling of the fatty tissues in real-time by taking a surface mesh and a few sample sequences of its physical behavior (Shi et al., 2008). Moreover, the method proposed in (Huang et al., 2006) is capable of synthesizing high resolution hand mesh deformation with rich and varying details, from only 14 examples poses. However, these approaches do not capture detailed soft-tissue deformations on a wide variety of body shapes. The major drawback of example-based methods is the need for example poses. Besides the fact that when the example poses cannot be captured on a real actor, creating these poses requires either tremendous effort from an artist, or a complex physical simulation on a volumetric version of the skin mesh. In both cases, the mesh and its associated skeleton at rest are not sufficient, and further human intervention is required. An interesting discussion on example-based deformation methods, can be found in (Feng et al., 2008). In the next section, we discuss several volume preservation methods for skinned characters, which have been proposed to tackle the loss of volume artefact of linear skinning techniques.

\section{VOLUME PRESERVING SKINNING METHODS}

Volume preservation is an important aspect in the context of skin deformation that has been addressed in a variety of research papers over the last years. Volume preservation methods allow artists to correct the volume changes through the generation of extra bulges and/or wrinkles. The method that has been proposed in (Desbrun and Gascuel, 1995) is one of the first methods to introduce volume preserving deformation, where they use local volume controllers to guarantee volume conservation of implicitly described soft substances. Moreover, multi-resolution methods (Guskov et al., 1999; Botsch and Kobbelt, 2003) can preserve surface details by decomposing a mesh into several frequency bands. Furthermore, Funck et al. presented an appealing approach that deforms the mesh vertices based on vector field integration (von Funck et al., 2006; von Funck et al., 2008). However, these two methods are either computationally expensive or do not fit into the standard animation pipeline. Angelidis and Singh developed a skinning algorithm based on a powerful embedding into the volumetric space, which enables to preserve volume locally and globally (Angelidis and Singh, 2007). In their method, a degree of freedom is left to the artist to control the final shape, although its combination with skinning weights variation along the mesh makes this control somewhat indirect. Recently, (Rohmer et al., 2008; Rohmer et al., 2009) presented an automatic volume correction method to model the constant volume behavior of soft tissues. It corrects the resultant deformations of LBS using a set of local deformations. In their work, they used an automatic way to segment an organic shape into a set of regions corresponding to the main muscle and fatty tissue areas, in which volume is computed and locally corrected. Huang et al. employed a nonlinear version of the volumetric graph Laplacian, which features nonlinear volume preservation constraints (Huang et al., 2006). Lipman et al. introduce a shape and volume preserving mesh editing technique (Lipman et al., 2007a), where meshes are represented by moving frames. These frames are scaled during deformation such that the volumetric shape properties are preserved.

Several impressive works that create an inner scaffolding of spring, which resist compression to maintain volume are described in (Hong et al., 2006; Zhou et al., 2005). The method in (Zhou et al., 2005) provides an excellent introduction to these interior lattice methods. Lattice-Based freeform deformation (FFD) are widely-used in commercial software (such as Autodesk 3D Studio Max and Maya) for providing smooth deformations and preserving the volume of the skin (Sederberg and Parry, 1986; Bloor and Wilson, 1990; Coquillart, 1990; Milliron et al., 2002). For example, Autodesk Maya 2007 supports the notion of flexors. This lattice flexor 


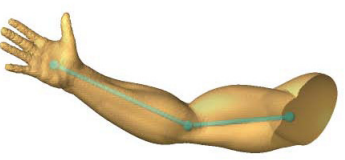

(a)

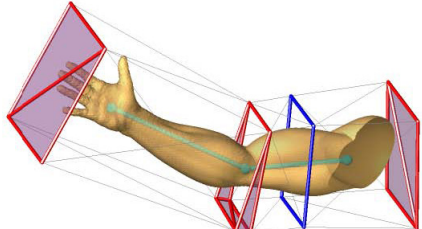

(b)

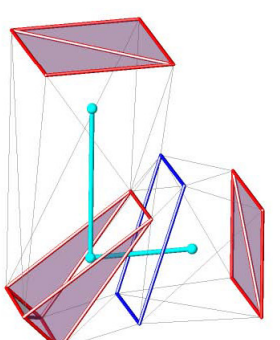

(c)

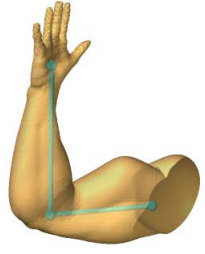

(d)

Figure 3: Skinning with cage: (a) Input geometry with skeleton. (b) An initial cage constructed from four templates, which are associated with the hand joint, elbow joint, upper arm bone, and the shoulder joint. (c) The skeleton deforms the mesh templates. (d) The geometry is deformed by the cage, yielding a non-pinching elbow and muscle bulging. (Image taken from (Ju et al., 2008).)

uses a local FFD lattice, which can then be driven by joint transformations. However, the flexors do not support skinning transfer and the use of flexors can require significant setup and tweaking because of the multitude of lattice points. FFD was first formally proposed in (Sederberg and Parry, 1986) both as a representation for free-form solids and as a method for sculpturing solid models. Using FFD, a complex character can be deformed by positioning the control vertices of the coarse control grid. A more general extension of FFD (EFFD) was later presented by (Coquillart, 1990). Although lattice-based methods give the artist the flexibility of creating the desired deformation, they require additional setup work and the deformation is sometimes difficult to predict.

On the other hand, cage-based skinning techniques consider an appealing way to control the deformation of an enclosed fine-detailed mesh and help to preserve the volume of skin deformations (Ju et al., 2005; Joshi et al., 2007; Lipman et al., 2007b; Ju et al., 2008; Savoye and Franco, 2010). Cage-based techniques can be considered as a generalization of the lattice-based freeform deformation. Instead of a regular control lattice, a cage is defined by a fixed-topology control lattice that is fitted to the character skin. The cage can be seen as a low-resolution abstraction of the character, which enables the user to deform a character using a simpler mesh. Most cage-based deformation methods are special case of linear blend skinning, where the handle (cage vertex) transformations are restricted to be translations and the focus is on choosing the weights. The method presented in (Ju et al., 2008) uses cage-based deformations to implement skinning templates, which offer a flexible design space within which to develop reusable skinning behavior. In their method, the skeleton drives the motion of the cage vertices using an example-based skinning technique, where the cage smoothly deforms the character model (see Fig. 3). Joshi et al. proposed a powerful cage-based deformation method based on harmonic coordinates (Joshi et al., 2007) for use in high-end character articulation. Their technique guarantees that the influence of each cage vertex is non-negative and falls off with distance as measured within the cage. It generates a pleasing deformation, but computing the harmonic coordinates is not easy. In spite of that, cage-based techniques allow smooth deformation of skin geometry. Posing the cage requires manual manipulation of the cage vertices. For an overview discussion on volume-preserving deformation methods, we refer the reader to (Nieto and Susin, 2013). Another promising way to preserve the volume of the skin and to achieve realistic deformation is by applying physics-based simulation into the skin layer around the skeleton. The following section describes the vast literature on physics-based methods.

\section{PHYSICS-BASED METHODS}

In order to model dynamic phenomena, such as the vibration of fatty tissues, muscles bulging and skin contact deformations due to collisions, the animator must configure the deformation for each keyframe. While manually posing a character for each animation keyframe allows artists to create such realistic effects, this process is tedious. Therefore, an alternative method is to employ physics into the skinning process, which highly enhances the believability and realism of character motions. Accordingly, physicsbased simulation manages to bring skeleton-driven animation beyond the purely kinematic approach by simulating secondary motions, such as jiggling of soft tissues when the character is moving. Those secondary motions enrich the visual experience of the animation and are essential for creating appealing characters for movie productions and virtual reality ap- 
plications. After the pioneering work of Terzopoulos et al. (Terzopoulos et al., 1987) and concurrently Lasseter's animation principle "squash and stretch" (Lasseter, 1987). Physical simulation has taken an important role in the animated feature film industry and computer games (Goktekin et al., 2007), where many physically based methods encouraged to simulate soft bodies or add dynamic effects to the skin. In the following subsections, we first discuss soft body simulations in (Section 4.1), and then physically based skinning methods in (Section 4.2).

\subsection{Deformable and Soft Bodies Simulations}

Simulating soft bodies can be achieved in different ways, and the design choice often has to balance the required accuracy and performance (Moore and Molloy, 2007). The most popular techniques for simulating soft bodies in computer animation are force-based methods. In particular, most of the techniques used to simulate dynamics rely on mass spring systems, because of the simplicity and efficiency. The general idea is to represent the vertices of the mesh as mass points, governed by Newton's second law of motion, and the edges as elastic massless links (spring). Hence, the mesh is deformed when the lengths of the elastic links change. This happens when the relative position of the mass points changes due to external forces. Mass-spring systems are based on a local description of the material, in which the physics of such systems is straightforward and the simulator is easy to implement. However, to simulate a particular material, it is important to select carefully the parameters of the springs, such as the stiffness and damping. Despite that these systems are fairly easy to implement, they suffer from instability and overshooting problems under large time steps. Moreover, mass-spring systems are often not accurate, since they are strongly topology dependent and are not built based on elasticity theory. On the other hand, finite element methods (FEM) allows to model elastic materials, in which both the masses and the internal and external forces are lumped to the vertices. The vertices in the mesh are treated like mass points in a mass spring system while each element acts like a generalized spring connecting all adjacent mass points. The methods presented in (Terzopoulos et al., 1987; Chadwick et al., 1989) are the first to demonstrate the effectiveness of comparatively simple mass-spring based approaches. In their methods, they applied the Lagrangian equations of motion using a finite difference scheme to simulate elastic objects with regular parametrizations. Here, the physical material properties can be described using only few parameters that are used to model soft bodies in an accurate manner. Unlike mass-spring systems, finite element methods are easy to simulate for any particular material. This makes things easier for artists in charge of modelling different types of soft bodies. Unfortunately, finite element methods are avoided in real-time applications, because they are computationally expensive and complex to implement. Various methods have been proposed to address the drawbacks of mass-spring systems and finite element methods (Gourret et al., 1989; Turner and Thalmann, 1993; Lee et al., 1995; Popović et al., 2003; Larboulette et al., 2005). A comprehensive survey of Nealen et al. (Nealen et al., 2006) provides the details about these techniques. The concept of employing dynamic simulations into skinning for the purpose of character animation was introduced over two decades ago (Girard and Maciejewski, 1985), where many techniques were proposed to reduce the accuracy of the simulation to help improve performance and interactivity. Capell et al. (Capell et al., 2002) used a volumetric finite element mesh to represent the deformation of skin, driven by the underlying skeleton motion. They extended their method to include rigging forces, which guide the deformation to a desired shape (Capell et al., 2005). In their method, they effectively handled the effect of skin movement by using skeletal constraints, but by using forces that can violate the conservation of momentum makes their simulation unstable under large time steps. Shinar et al. (Shinar et al., 2008) presented a framework of a two-way coupling between rigid and deformable bodies, in which they use a time integration scheme for solving dynamic elastic deformations in soft bodies interacting with rigid bodies. However, their method does not facilitate the development of an interactive animation system, because of the massive computation required for the finite elements representing the deformable body. In contrast, a possible way to accelerate the simulation of soft bodies is to focus on the surface rather than the volume (Bro-nielsen and Cotin, 1996). In particular, Galoppo et al. (Galoppo et al., 2007) presented a fast method to compute the skin deformation on the surface of a soft body with rigid core. Their formulation only considers the elastic energy from skin-layer deformation, and does not include the deformation inside the volume. This may lead to inaccuracies when capturing pose-dependent deformations. All the above methods are only valid for small deformations and are unsuitable for an articulated character's large deformations. On the other hand, Müller et al. (Müller et al., 2002) achieved a good real-time 

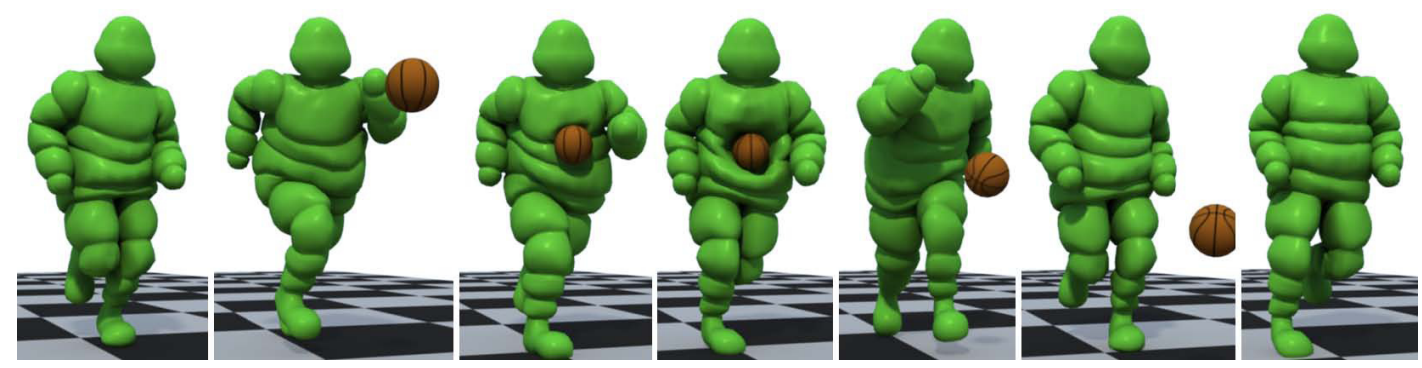

Figure 4: Employing dynamic simulation into skinning process allows two-way interactions between the skeleton, the skin geometry, and the environment at interactive rates. (Image taken from (Liu et al., 2013).)

performances for large rotational deformations, by using a pre-computed linear stiffness matrix to generate the deformations; their method is simple and rotationally invariant. Recently, Kim and Pollard (Kim and Pollard, 2011) proposed an approach relying on finite element method to simulate the skin deformation, able to handle both one-way and twoway simulations. Their method generates compelling dynamic effects and the deformations are obtained at near interactive rate. The method proposed in (Gilles et al., 2011) simulates dynamic skinned deformation models using frame-based degrees of freedom with unreduced force evaluation. Jain and Liu presented a robust approach that realistically simulate characters with soft tissue at the site of contact, where they used two-way coupling between articulated rigid bodies and deformable objects (Jain and Liu, 2011). Liu et al. developed a framework that simulates and controls skeleton driven soft body characters (Liu et al., 2013). Their method couples the skeleton dynamics and the soft body dynamics to enable two way interactions between the skeleton, the skin geometry, and the environment at interactive rates (as we can see in Fig. 4).

\subsection{Physically based Skinning}

Physics-based methods are the natural choice for creating secondary motion effects such as flesh jiggling when a character is moving (Turner and Thalmann, 1993; Lee et al., 2009; Gilles et al., 2011; Saito et al., 2015). Turner and Thalmann model the elasticity of skin for character animation and simulate the fat layer by Hookian spring forces (Turner and Thalmann, 1993). However, they treat muscles as purely controlled elements. Thus, they do not model muscles with deformable methods. Wilhelms (Wilhelms, 1994) presented an approach for animated animals by simulating individual bones, muscles, soft tissues and skin. The use of muscles, soft tissues and flesh elements makes it hard to fit this approach into the skinning framework. Moreover, Hahn et al. (Hahn et al., 2012; Hahn et al., 2013) generated secondary skin dynamics based on the rig degrees of freedom. Their methods simulate the deformation of a character's fat and muscles in the nonlinear subspace induced by the character rig. In the other direction, Kim and James (Kim and James, 2011) proposed a domain-decomposition method to simulate articulated deformable characters entirely within a subspace framework, where they combined locally rotated nonlinear subspace models to simulate the detailed deformations of the models. In order to simulate the musculotendons of the human hand and forearm, (Sueda et al., 2008) add anatomic detail using the tendons and bones. While physics-based skinning methods can automatically generate secondary motion with high visual quality, they entail a significant computational burden that slows production and prohibits its use in interactive environments. McAdams et al. (McAdams et al., 2011) presented a robust method using a uniform hexahedral lattice, which provides convincing deformations of the skin with contact handling. In addition, they introduce a onepoint quadrature scheme and a multi-grid solver in order to improve the performance and stabilize the simulation. Although their method can capture appealing skin deformations and guarantee pinch-free geometry, it works at best at near interactive performance (as we can see in Fig. 5). Recently, Deul and Bender (Bender et al., 2013) introduced a multi-layer character skinning based on shape matching with oriented particles, used to simulate the elastic behavior of a closed triangular mesh as a representation of a skin model. They make a use of position-based constraints for coupling the skeleton with the skin and handling self-collisions. In (Rumman and Fratarcangeli, 2014; Rumman et al., 2015; Abu Rumman and Fratarcangeli, 2015), a two-layered approach, the skin is first deformed with a classic linear blend skinning and then the vertex positions are adjusted using Position-based Dynamics. This allows to robustly mimic the behavior of the skin, and achieve and tune 

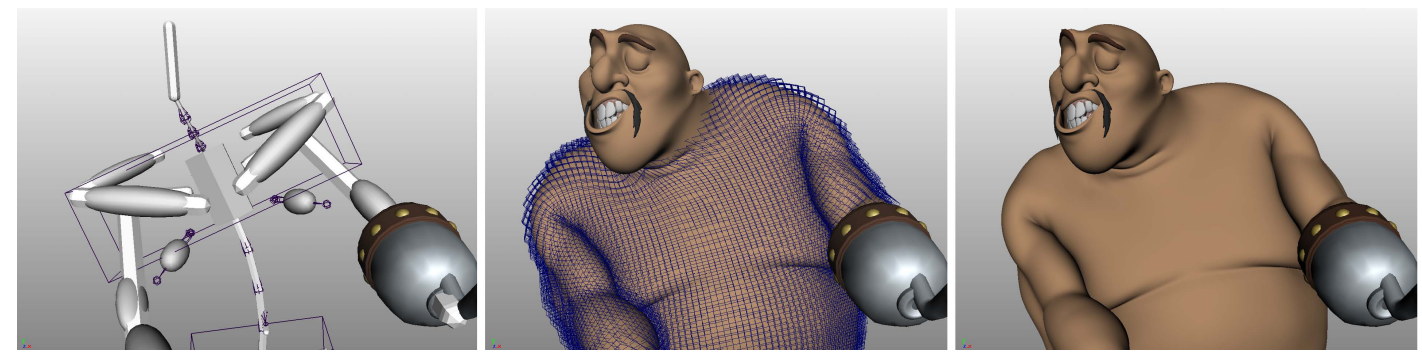

Figure 5: The Method of (McAdams et al., 2011) takes a skeleton and a surface mesh as input. Based on a hexahedral lattice with 106,567 cells (center), their method simulates the deformed surface (right) obeying self-collision and volumetric elasticity at 5.5 seconds per frame. (Image taken from (McAdams et al., 2011).)

effects like volume preservation and jiggling at interactive rate. Lastly, Gao et al. (Gao et al., 2014) proposed a physics-based skinning method for skeletonbased characters. They introduced a material model of ex-rotated elasticity, which uses a procedural skinning technique to approximate co-rotated elasticity with an affine force model and pose-dependent coefficients. Their method can simulate high-resolution human flesh models with full external and self-collision processing, without the ability to generate secondary motions effects like jiggling of the fat tissues.

\section{Conclusion}

In this paper, we have reviewed the common techniques for modelling deformations, especially those for character animation purposes. A greater attention was paid to skeleton-based methods, and physicsbased methods. In skeleton-based skinning such as linear blend skinning, dual quaternion skinning and pose space deformation, surface deformation is restricted to the skeletal pose that fully defines the surface deformation. While skeleton-based methods can produce good results, the believability of the deformation using these methods is limited. Because they cannot capture secondary motions effects and skin contact deformations. Skeleton-based deformation alone is not sufficient for capturing believable skin deformations, such as skin stretching, secondary motion effects and skin contact due to collisions. In contrast, physics-based simulations bring skeleton-based animation beyond the purely kinematic approach by simulating secondary motions such as jiggling of soft tissues when the character is moving, as well as capturing skin contact deformation. Simulating such fleshlike deformations is difficult due to the coupling between the skeleton and soft skin. Moreover, the resultant deformation has a high number of independent degrees of freedom, in which it does not respect any manipulation done by the artist. Therefore, once the deformation parameters are specified, it is difficult to control the actual resulting shape of the character in every animation frame. Furthermore, physicsbased methods are computationally expensive and usually avoided in interactive applications. In the future skinning research, the computational process for obtaining believable skin motion must trade-off between these requirements: it must (1) be fast enough to achieve interactive rate (i.e., $>30 \mathrm{fps}$ ), (2) produce believable animation to minimize manual postprocessing time, and (3) be controllable and stable.

\section{REFERENCES}

Abu Rumman, N. and Fratarcangeli, M. (2015). Position-based skinning for soft articulated characters. Computer Graphics Forum, 34(6):240250 .

Alexa, M. (2002). Linear combination of transformations. In Proceedings of the 29th Annual Conference on Computer Graphics and Interactive Techniques, SIGGRAPH '02, pages 380-387, New York, NY, USA. ACM.

Allen, B., Curless, B., and Popović, Z. (2002). Articulated body deformation from range scan data. In Proceedings of the 29th Annual Conference on Computer Graphics and Interactive Techniques, SIGGRAPH '02, pages 612-619, New York, NY, USA. ACM

Angelidis, A. and Singh, K. (2007). Kinodynamic skinning using volumepreserving deformations. In Proceedings of the 2007 ACM SIGGRAPH/Eurographics Symposium on Computer Animation, SCA 2007, San Diego, California, USA, August 2-4, 2007, pages 129-140.

Baran, I. and Popović, J. (2007). Automatic rigging and animation of 3d characters. In ACM SIGGRAPH 2007 Papers, SIGGRAPH '07, New York, NY, USA. ACM.

Bender, J., Müller, M., Otaduy, M. A., and Teschner, M. (2013). Positionbased methods for the simulation of solid objects in computer graphics. In EUROGRAPHICS 2013 State of the Art Reports. Eurographics Association.

Bharaj, G., Thormählen, T., Seidel, H.-P., and Theobalt, C. (2012). Automatically rigging multi-component characters. Comp. Graph. Forum, 31(2pt3):755-764

Bloor, M. I. G. and Wilson, M. J. (1990). Using partial differential equations to generate free-form surfaces: 91787. Comput. Aided Des. 22(4):202-212.

Botsch, M. and Kobbelt, L. (2003). Multiresolution surface representation based on displacement volumes. Computer Graphics Forum, 22(3):483-491.

Bro-nielsen, M. and Cotin, S. (1996). Real-time volumetric deformable models for surgery simulation using finite elements and condensation. In Computer Graphics Forum, pages 57-66. 
Capell, S., Burkhart, M., Curless, B., Duchamp, T., and Popović, Z. (2005). Physically based rigging for deformable characters. In Proceedings of the 2005 ACM SIGGRAPH/Eurographics Symposium on Computer Animation, SCA '05, pages 301-310, New York, NY, USA. ACM

Capell, S., Green, S., Curless, B., Duchamp, T., and Popović, Z. (2002). Interactive skeleton-driven dynamic deformations. In Proceedings of the 29th Annual Conference on Computer Graphics and Interactive Techniques, SIGGRAPH '02, pages 586-593, New York, NY, USA. ACM.

Chadwick, J. E., Haumann, D. R., and Parent, R. E. (1989). Layered construction for deformable animated characters. SIGGRAPH Comput. Graph., 23(3):243-252.

Chen, C.-H., Lin, I.-C., Tsai, M.-H., and Lu, P.-H. (2011). Lattice-based skinning and deformation for real-time skeleton-driven animation. In Proceedings of the 2011 12th International Conference on ComputerAided Design and Computer Graphics, CADGRAPHICS '11, pages 306-312, Washington, DC, USA. IEEE Computer Society.

Coquillart, S. (1990). Extended free-form deformation: A sculpturing tool for 3d geometric modeling. In Proceedings of the 17th Annual Conference on Computer Graphics and Interactive Techniques, SIGGRAPH '90, pages 187-196, New York, NY, USA. ACM.

Desbrun, M. and Gascuel, M.-P. (1995). Animating soft substances with implicit surfaces. In Proceedings of the 22Nd Annual Conference on Computer Graphics and Interactive Techniques, SIGGRAPH '95, pages 287-290, New York, NY, USA. ACM.

Dionne, O. and de Lasa, M. (2013). Geodesic voxel binding for production character meshes. In Proceedings of the 12th ACM SIGGRAPH/Eurographics Symposium on Computer Animation, SCA '13, pages 173-180, New York, NY, USA. ACM.

Feng, W.-W., Kim, B.-U., and Yu, Y. (2008). Real-time data driven deformation using kernel canonical correlation analysis. In $A C M$ SIGGRAPH 2008 Papers, SIGGRAPH '08, pages 91:1-91:9, New York, NY, USA. ACM.

Forstmann, S. and Ohya, J. (2006). Fast skeletal animation by skinned arcspline based deformation. EG 2006 Short Papers, pages 1-4.

Forstmann, S., Ohya, J., Krohn-Grimberghe, A., and McDougall, R. (2007) Deformation styles for spline-based skeletal animation. In Proceedings of the 2007 ACM SIGGRAPH/Eurographics Symposium on Computer Animation, SCA '07, pages 141-150, Aire-la-Ville, Switzerland, Switzerland. Eurographics Association.

Galoppo, N., Otaduy, M. A., Tekin, S., Gross, M. H., and Lin, M. C. (2007). Soft articulated characters with fast contact handling. Comput. Graph. Forum, 26(3):243-253.

Gao, M., Mitchell, N., and Sifakis, E. (2014). Steklov-poincaré skinning. In The Eurographics / ACM SIGGRAPH Symposium on Computer Animation, SCA '14, Copenhagen, Denmark, 2014., pages 139-148.

Gilles, B., Bousquet, G., Faure, F., and Pai, D. K. (2011). Frame-based elastic models. ACM Trans. Graph., 30(2):15:1-15:12.

Girard, M. and Maciejewski, A. A. (1985). Computational modeling for the computer animation of legged figures. In Proceedings of the 12th Annual Conference on Computer Graphics and Interactive Techniques, SIGGRAPH '85, pages 263-270, New York, NY, USA. ACM.

Goktekin, T. G., Reisch, J., Peachey, D., and Shah, A. (2007). An effects recipe for rolling a dough, cracking an egg and pouring a sauce. In ACM SIGGRAPH 2007 Sketches, SIGGRAPH '07, New York, NY, USA. ACM.

Gourret, J.-P., Thalmann, N. M., and Thalmann, D. (1989). Simulation of object and human skin formations in a grasping task. In Proceedings of the 16th Annual Conference on Computer Graphics and Interac- tive Techniques, SIGGRAPH '89, pages 21-30, New York, NY, USA. ACM.

Guskov, I., Sweldens, W., and Schröder, P. (1999). Multiresolution signal processing for meshes. In Proceedings of the 26th Annual Conference on Computer Graphics and Interactive Techniques, SIGGRAPH '99, pages 325-334, New York, NY, USA. ACM Press/Addison-Wesley Publishing Co.

Hahn, F., Martin, S., Thomaszewski, B., Sumner, R., Coros, S., and Gross, M. (2012). Rig-space physics. ACM Trans. Graph., 31(4):72:1-72:8.

Hahn, F., Thomaszewski, B., Coros, S., Sumner, R., and Gross, M. (2013) Efficient simulation of secondary motion in rig-space. In Proceedings of the ACM SIGGRAPH/Eurographics Symposium on Computer Animation, SCA '13.

Hejl, J. (2004). Hardware skinning with quaternions. In Kirmse, A., editor, Game Programming Gems 4, pages 487-495. Charles River Media.

Hong, M., Jung, S., Choi, M.-H., and Welch, S. (2006). Fast volume preservation for a mass-spring system. Computer Graphics and Applications, IEEE, 26(5):83-91.

Huang, J., Shi, X., Liu, X., Zhou, K., Wei, L.-Y., Teng, S.-H., Bao, H., Guo, B., and Shum, H.-Y. (2006). Subspace gradient domain mesh deformation. ACM Trans. Graph., 25(3):1126-1134.

Jacka, D., Reid, A., Merry, B., and Gain, J. (2007). A comparison of linear skinning techniques for character animation. In Proceedings of the 5th International Conference on Computer Graphics, Virtual Reality, Visualisation and Interaction in Africa, AFRIGRAPH '07, pages 177-186, New York, NY, USA. ACM

Jacobson, A., Baran, I., Kavan, L., Popović, J., and Sorkine, O. (2012). Fast automatic skinning transformations. ACM Trans. Graph., 31(4):77:177:10.

Jacobson, A., Baran, I., Popović, J., and Sorkine, O. (2011). Bounded biharmonic weights for real-time deformation. ACM Trans. Graph., 30(4):78:1-78:8

Jacobson, A., Deng, Z., Kavan, L., and Lewis, J. (2014). Skinning: Real-time shape deformation. In ACM SIGGRAPH 2014 Courses.

Jacobson, A. and Sorkine, O. (2011). Stretchable and twistable bones for skeletal shape deformation. In Proceedings of the 2011 SIGGRAPH Asia Conference, SA '11, pages 165:1-165:8, New York, NY, USA. ACM.

Jain, S. and Liu, C. K. (2011). Controlling physics-based characters using soft contacts. ACM Trans. Graph. (SIGGRAPH Asia), 30:163:1163:10

Joshi, P., Meyer, M., DeRose, T., Green, B., and Sanocki, T. (2007). Harmonic coordinates for character articulation. ACM Trans. Graph., 26(3).

Ju, T., Schaefer, S., and Warren, J. (2005). Mean value coordinates for closed triangular meshes. ACM Trans. Graph., 24(3):561-566.

Ju, T., Zhou, Q.-Y., van de Panne, M., Cohen-Or, D., and Neumann, U. (2008). Reusable skinning templates using cage-based deformations. ACM Trans. Graph., 27(5):122:1-122:10.

Kavan, L., Collins, S., and O'Sullivan, C. (2009). Automatic linearization of nonlinear skinning. In Proceedings of the 2009 Symposium on Interactive 3D Graphics and Games, I3D '09, pages 49-56, New York, NY, USA. ACM.

Kavan, L., Collins, S., Zára, J., and O’Sullivan, C. (2007). Skinning with dual quaternions. In Proceedings of the 2007 Symposium on Interactive $3 D$ Graphics and Games, I3D '07, pages 39-46, New York, NY, USA. ACM.

Kavan, L. and Sorkine, O. (2012). Elasticity-inspired deformers for character articulation. ACM Trans. Graph., 31(6):196:1-196:8. 
Kavan, L. and Zára, J. (2005). Spherical blend skinning: A real-time deformation of articulated models. In Proceedings of the 2005 Symposium on Interactive 3D Graphics and Games, I3D '05, pages 9-16, New York, NY, USA. ACM.

Kim, J. and Pollard, N. S. (2011). Fast simulation of skeleton-driven deformable body characters. ACM Trans. Graph., 30(5):121:1-121:19.

Kim, T. and James, D. L. (2011). Physics-based character skinning using multi-domain subspace deformations. In Proceedings of the 2011 ACM SIGGRAPH/Eurographics Symposium on Computer Animation, SCA '11, pages 63-72, New York, NY, USA. ACM.

Kim, Y. and Han, J. (2014). Bulging-free dual quaternion skinning. Journal of Visualization and Computer Animation, 25(3-4):323-331.

Kry, P. G., James, D. L., and Pai, D. K. (2002). Eigenskin: Real time large deformation character skinning in hardware. In Proceedings of the 2002 ACM SIGGRAPH/Eurographics Symposium on Computer Animation, SCA '02, pages 153-159, New York, NY, USA. ACM.

Kurihara, T. and Miyata, N. (2004). Modeling deformable human hands from medical images. In Proceedings of the 2004 ACM SIGGRAPH/Eurographics Symposium on Computer Animation, SCA '04, pages 355-363, Aire-la-Ville, Switzerland, Switzerland. Eurographics Association.

Larboulette, C., Cani, M.-P., and Arnaldi, B. (2005). Dynamic skinning: adding real-time dynamic effects to an existing character animation. In $S C C G$, pages $87-93$.

Lasseter, J. (1987). Principles of traditional animation applied to 3d computer animation. SIGGRAPH Comput. Graph., 21(4):35-44.

Le, B. H. and Deng, Z. (2012). Smooth skinning decomposition with rigid bones. ACM Trans. Graph., 31(6):199:1-199:10.

Le, B. H. and Deng, Z. (2014). Robust and accurate skeletal rigging from mesh sequences. ACM Trans. Graph., 33(4):84:1-84:10.

Lee, G. S., Lin, A., Schiller, M., Peters, S., McLaughlin, M., and Hanner, F. (2013). Enhanced dual quaternion skinning for production use. In ACM SIGGRAPH 2013 Talks, SIGGRAPH '13, pages 9:1-9:1, New York, NY, USA. ACM.

Lee, S.-H., Sifakis, E., and Terzopoulos, D. (2009). Comprehensive biomechanical modeling and simulation of the upper body. ACM Trans. Graph., 28(4):99:1-99:17.

Lee, Y., Terzopoulos, D., and Waters, K. (1995). Realistic modeling for facial animation. In Proceedings of the 22Nd Annual Conference on Computer Graphics and Interactive Techniques, SIGGRAPH '95, pages 55-62, New York, NY, USA. ACM.

Lewis, J. P., Cordner, M., and Fong, N. (2000). Pose space deformation: A unified approach to shape interpolation and skeleton-driven deformation. In Proceedings of the 27th Annual Conference on Computer Graphics and Interactive Techniques, SIGGRAPH '00, pages 165172, New York, NY, USA. ACM Press/Addison-Wesley Publishing Co.

Lipman, Y., Cohen-Or, D., Gal, R., and Levin, D. (2007a). Volume and shape preservation via moving frame manipulation. ACM Trans. Graph., 26(1).

Lipman, Y., Kopf, J., Cohen-Or, D., and Levin, D. (2007b). Gpu-assisted positive mean value coordinates for mesh deformations. In Proceedings of the Fifth Eurographics Symposium on Geometry Processing, SGP '07, pages 117-123, Aire-la-Ville, Switzerland, Switzerland. Eurographics Association.

Liu, L., Yin, K., Wang, B., and Guo, B. (2013). Simulation and control of skeleton-driven soft body characters. ACM Trans. Graph., 32(6):215:1-215:8.
Magnenat-Thalmann, N., Laperrière, R., and Thalmann, D. (1988). Jointdependent local deformations for hand animation and object grasping. In Proceedings on Graphics Interface '88, pages 26-33, Toronto, Ont., Canada. Canadian Information Processing Society

McAdams, A., Zhu, Y., Selle, A., Empey, M., Tamstorf, R., Teran, J., and Sifakis, E. (2011). Efficient elasticity for character skinning with contact and collisions. ACM Trans. Graph., 30(4):37:1-37:12.

Merry, B., Marais, P., and Gain, J. (2006). Animation space: A truly linear framework for character animation. ACM Trans. Graph., 25(4):14001423

Milliron, T., Jensen, R. J., Barzel, R., and Finkelstein, A. (2002). A framework for geometric warps and deformations. ACM Trans. Graph. 21(1):20-51.

Min, K.-H., Baek, S.-M., Lee, G. A., Choi, H., and Park, C.-M. (2000). Anatomically-based modeling and animation of human upper limbs. In Proceedings of International Conference on Human Modeling and Animation.

Mohr, A. and Gleicher, M. (2003). Building efficient, accurate character skins from examples. In ACM SIGGRAPH 2003 Papers, SIGGRAPH '03, pages 562-568, New York, NY, USA. ACM.

Moore, P. and Molloy, D. (2007). A survey of computer-based deformable models. In Machine Vision and Image Processing Conference, 2007. IMVIP 2007. International, pages 55-66.

Müller, M., Dorsey, J., McMillan, L., Jagnow, R., and Cutler, B. (2002). Stable real-time deformations. In Proceedings of the 2002 ACM SIGGRAPH/Eurographics Symposium on Computer Animation, pages 49-54, New York, NY, USA. ACM.

Nealen, A., Mueller, M., Keiser, R., Boxerman, E., and Carlson, M. (2006). Physically Based Deformable Models in Computer Graphics. Computer Graphics Forum, 25(4):809-836.

Nieto, J. and Susin, A. (2013). Cage based deformations: A survey. In Deformation Models, volume 7 of Lecture Notes in Computational Vision and Biomechanics, pages 75-99. Springer Netherlands.

Park, S. I. and Hodgins, J. K. (2006). Capturing and animating skin deformation in human motion. In ACM SIGGRAPH 2006 Papers, SIGGRAPH '06, pages 881-889, New York, NY, USA. ACM.

Park, S. I. and Hodgins, J. K. (2008). Data-driven modeling of skin and muscle deformation. In ACM SIGGRAPH 2008 Papers, SIGGRAPH '08, pages 96:1-96:6, New York, NY, USA. ACM.

Popović, J., Seitz, S. M., and Erdmann, M. (2003). Motion sketching for control of rigid-body simulations. ACM Trans. Graph., 22(4):10341054

Rhee, T., Lewis, J. P., and Neumann, U. (2006). Real-time weighted posespace deformation on the GPU. Comput. Graph. Forum, 25(3):439448 .

Rohmer, D., Hahmann, S., and Cani, M. (2008). Local volume preservation for skinned characters. Comput. Graph. Forum, 27(7):1919-1927.

Rohmer, D., Hahmann, S., and Cani, M.-P. (2009). Exact volume preserving skinning with shape control. In Proceedings of the 2009 ACM SIGGRAPH/Eurographics Symposium on Computer Animation, SCA '09, pages 83-92, New York, NY, USA. ACM.

Rumman, N. A. and Fratarcangeli, M. (2014). Position based skinning of skeleton-driven deformable characters. In Proceedings of the 30th Spring Conference on Computer Graphics, SCCG '14, pages 83-90, New York, NY, USA. ACM.

Rumman, N. A., Schaerf, M., and Bechmann, D. (2015). Collision detection for articulated deformable characters. In Proceedings of the 8th ACM SIGGRAPH Conference on Motion in Games, MIG '15, pages 215220, New York, NY, USA. ACM 
Saito, S., Zhou, Z.-Y., and Kavan, L. (2015). Computational bodybuilding: Anatomically-based modeling of human bodies. ACM Trans. Graph., 34(4):41:1-41:12.

Savoye, Y. and Franco, J.-S. (2010). Cageik: Dual-laplacian cage-based inverse kinematics. In Proceedings of the 6th International Conference on Articulated Motion and Deformable Objects, AMDO'10, pages 280-289, Berlin, Heidelberg. Springer-Verlag.

Schumacher, C., Thomaszewski, B., Coros, S., Martin, S., Sumner, R., and Gross, M. (2012). Efficient simulation of example-based materials. In Proceedings of the ACM SIGGRAPH/Eurographics Symposium on Computer Animation, SCA '12, pages 1-8, Aire-la-Ville, Switzerland, Switzerland. Eurographics Association.

Sederberg, T. W. and Parry, S. R. (1986). Free-form deformation of solid geometric models. In Proceedings of the 13th Annual Conference on Computer Graphics and Interactive Techniques, SIGGRAPH '86, pages 151-160, New York, NY, USA. ACM.

Shi, X., Zhou, K., Tong, Y., Desbrun, M., Bao, H., and Guo, B. (2008). Example-based dynamic skinning in real time. ACM Trans. Graph., 27(3):29:1-29:8

Shinar, T., Schroeder, C., and Fedkiw, R. (2008). Two-way coupling of rigid and deformable bodies. In Proceedings of the 2008 ACM SIGGRAPH/Eurographics Symposium on Computer Animation, SCA '08, pages 95-103, Aire-la-Ville, Switzerland, Switzerland. Eurographics Association.

Sloan, P.-P. J., Rose, III, C. F., and Cohen, M. F. (2001). Shape by example. In Proceedings of the 2001 Symposium on Interactive 3D Graphics, I3D '01, pages 135-143, New York, NY, USA. ACM.

Sueda, S., Kaufman, A., and Pai, D. K. (2008). Musculotendon simulation for hand animation. ACM Trans. Graph., 27(3):83:1-83:8.

Terzopoulos, D., Platt, J., Barr, A., and Fleischer, K. (1987). Elastically deformable models. SIGGRAPH Comput. Graph., 21(4):205-214.

Turner, R. and Thalmann, D. (1993). The elastic surface layer model for animated character construction. In PROCEEDINGS OF COMPUTER GRAPHICS INTERNATIONAL '93, pages 399-412. SpringerVerlag.

Vaillant, R., Barthe, L., Guennebaud, G., Cani, M.-P., Rohmer, D., Wyvill, B., Gourmel, O., and Paulin, M. (2013). Implicit skinning: Realtime skin deformation with contact modeling. ACM Trans. Graph., 32(4):125:1-125:12

Vaillant, R., Guennebaud, G., Barthe, L., Wyvill, B., and Cani, M.-P. (2014). Robust iso-surface tracking for interactive character skinning. ACM Trans. Graph., 33(6):189:1-189:11.

von Funck, W., Theisel, H., and Seidel, H. (2008). Volume-preserving mesh skinning. In Proceedings of the Vision, Modeling, and Visualization Conference 2008, VMV 2008, Konstanz, Germany, October 8-10, 2008, pages 409-414.

von Funck, W., Theisel, H., and Seidel, H.-P. (2006). Vector field based shape deformations. ACM Trans. Graph., 25(3):1118-1125.

Wang, R. Y., Pulli, K., and Popović, J. (2007). Real-time enveloping with rotational regression. ACM Trans. Graph., 26(3).

Wang, X. C. and Phillips, C. (2002). Multi-weight enveloping: Least-squares approximation techniques for skin animation. In Proceedings of the 2002 ACM SIGGRAPH/Eurographics Symposium on Computer Animation, SCA '02, pages 129-138, New York, NY, USA. ACM.

Wilhelms, J. (1994). Modeling animals with bones, muscles, and skin. Technical report, University of California.

Yang, X., Somasekharan, A., and Zhang, J. J. (2006). Curve skeleton skinning for human and creature characters. Computer Animation and Virtual Worlds, 17(3-4):281-292.
Zhou, K., Huang, J., Snyder, J., Liu, X., Bao, H., Guo, B., and Shum, H.-Y. (2005). Large mesh deformation using the volumetric graph laplacian. In ACM SIGGRAPH 2005 Papers, SIGGRAPH '05, pages 496-503, New York, NY, USA. ACM. 